\title{
Ensino de Lógica através de estratégias de Demonstração e Refutação: A integração do tutorial interativo TryLogic via IMS Learning Tools Interoperability
}

\author{
Patrick Terrematte ${ }^{1}$, João Marcos ${ }^{2}$ \\ ${ }^{1}$ Departamento de Filosofia (DFil/CCHLA) \\ ${ }^{2}$ Departamento de Informática e Matemática Aplicada (DIMAp/CCET) \\ Universidade Federal do Rio Grande do Norte (UFRN) - Natal - RN - Brasil \\ terrematte@ppgsc.ufrn.br, jmarcos@dimap.ufrn.br
}

\begin{abstract}
The present study proposes an interactive tutorial, namely the TRYLOGIC, aimed at teaching to solve logical conjectures either by proofs or refutations. The study also aims at: describing the integration of our infrastructure with the Virtual Learning Environment Moodle through the IMS Learning Tools Interoperability specification; presenting the Conjecture Generator that works for the tasks involving proving and refuting; and, finally evaluating the tool.
\end{abstract}

\section{Introdução}

A Lógica é uma disciplina basilar para a Computação, todavia a dificuldade dos alunos ao aprenderem e apreciarem a relevância das habilidades lógicas sugere na realidade um problema curricular, sobretudo com o papel da Lógica na estrutura dos cursos e na metodologia de apresentação tradicional. A solução proposta por [Barland et al. 2000, p.4] é integrar o ensino de lógica com a utilização de ferramentas no contexto da aprendizagem para assimilar conceitos abstratos através de exemplos concretos. Anteriormente, isto nos motivou a elaborar uma metodologia de ensino para guiar o aluno em tarefas que combinam características do ensino presencial tradicional com recursos da educação à distância [Terrematte et al. 2011]. Nosso trabalho aqui é resultado de uma estratégia de ensino da Lógica para resolver tarefas de demonstrar ou refutar, e da criação de um tutorial interativo para guiar o aluno de forma gradual e sequencial: o TRYLOGIC ${ }^{1}$.

\section{Metodologia}

A nossa metodologia durante a preparação do trabalho foi implementada através da investigação do estado da arte das ferramentas de ensino de Lógica. Para a construção de nossa infraestrutura optamos por utilizar e integrar as seguintes ferramentas:

- ProofWeb²: uma interface web para assistentes de demonstração de teoremas [Hendriks et al. 2010].

- Coq: um assistente de demonstração de teoremas em teorias formais.

- Gerador de Conjecturas: uma ferramenta de geração de exercícios.

- Moodle: um ambiente virtual de aprendizagem para acompanhamento de alunos.

Nossos objetivos específicos envolveram a integração das ferramentas descritas acima através da especificação IMS Learning Tools Interoperability (IMS LTI) e a implementação de uma teoria no Coq para refutar conjecturas em Lógica Proposicional.

\footnotetext{
${ }^{1}$ Disponível para acesso através do endereço http://lolita.dimap.ufrn.br/trylogic.

${ }^{2}$ Disponível originalmente para acesso através do endereço http://prover.cs.ru.nl/
} 


\section{Lógica Proposicional para Demonstrar e Refutar}

A Lógica Proposicional $\left(\mathrm{L}_{P r o p}\right)$ é a parte da lógica que trata de proposições declarativas, ou seja, afirmações que podem ser verdadeiras ou falsas, e expressas através de conectivos lógicos e das regras que os manipulam. Sua importância se deve ao fato de que muitos conceitos complexos e sofisticados de lógica matemática aparecem primariamente aqui num contexto mais simples. Todas as sentenças proposicionais podem ser formadas composicionalmente a partir dos seguintes conectivos lógicos (com sua aridade indicada no índice), respectivamente: a conjunção $\left(\wedge_{2}\right)$, a disjunção $\left(\vee_{2}\right)$, a implicação $\left(\rightarrow_{2}\right)$, a biimplicação $\left(\leftrightarrow_{2}\right)$, a negação $\left(\neg_{1}\right)$ e o absurdo $\left(\perp_{0}\right)$.

A linguagem da lógica proposicional é definida recursivamente, como se segue, considere um conjunto de letras proposicionais Prop $=\left\{A, B, C, D, E, F, A_{0}, B_{0}, C_{0}\right.$, $\left.D_{0}, E_{0}, F_{0}, A_{1}, B_{1}, C_{1}, D_{1}, E_{1}, F_{1} \ldots\right\}$; um conjunto de conectivos Conec $=\left\{\perp_{0}, \neg_{1}, \rightarrow_{2}\right.$, $\leftrightarrow_{2}, \wedge_{2}$ e $\left.\vee_{2}\right\}$; um alfabeto proposicional $\Sigma=$ Prop $\cup$ Conec $\cup\{()$,$\} ; e esquemas de$ fórmulas construídas através de letras do proposicionais representadas pelas letras gregas $(\alpha, \beta, \gamma, \delta, \epsilon, \phi, \psi \ldots)$ e denotam meta-fórmulas que podem ser substituídas por instâncias da meta-fórmula.

Se desejarmos demonstrar conjecturas — como a asserção $\Gamma \vdash \alpha$ segundo a que a partir de um conjunto $\Gamma$ de fórmulas é derivada uma fórmula $\alpha-$, é preciso tentar construir uma árvore de derivação usando as regras de dedução da teoria $N_{p}$, que no caso da conjunção, são expressas pelas seguintes regras:

$$
\frac{\alpha \beta}{\alpha \wedge \beta}(\wedge I) \quad \frac{\alpha \wedge \beta}{\alpha}\left(\wedge E_{1}\right) \quad \frac{\alpha \wedge \beta}{\beta}\left(\wedge E_{2}\right)
$$

Por outro lado, para refutar conjecturas - como no caso da asserção $\Gamma \not \models \alpha$ segundo a qual existe uma valoração $v$ que satisfaz $\Gamma$, mas falsifica $\alpha-$, é preciso construir árvores de refutação na Teoria $\mathrm{Sem}_{p}$, por exemplo, aplicando algumas das seguintes regras/táticas da conjunção:

$$
\frac{v \Vdash \alpha \quad v \Vdash \beta}{v \Vdash \alpha \wedge \beta}(\wedge T) \quad \frac{v \nVdash \alpha}{v \nVdash \alpha \wedge \beta}(\wedge F 1) \quad \frac{v \nVdash \beta}{v \nVdash \alpha \wedge \beta}(\wedge F 2)
$$

Através destas e de outras regras, nós implementamos a teoria $\mathrm{Sem}_{p}$ no ProofWeb e podemos mostrar que uma dada sentença não é consequência semântica de um certo conjunto de sentenças dadas. De forma diferente do sistema $N_{p}$, as regras de inferência envolvem a manipulação das asserções sobre a interpretação de fórmulas satisfeitas ou falsificadas por uma valoração $v$.

Neste sistema uma refutação de $(\neg \mathrm{A} \rightarrow \mathrm{B}) \wedge(\neg \mathrm{A} \wedge \neg \mathrm{B})$ é produzida pela exibição, acompanhada da respectiva justificativa de que existe pelo menos uma valoração $v$ que falsifica tal sentença. Uma das vantagens didáticas do TRYLOGIC, em conjunto com o ProofWeb, é sua coerência com ao Princípio de Representação Múltipla (The Modality Effect) da Teoria da Carga Cognitiva [Sweller et al. 2011, p.129]. Com a Figura 1 podemos observar que sem a sequência de táticas inserida no lado esquerdo (a) não seria possível verificar o objeto apresentado no lado direito (b), pois a sequência de táticas representa uma justificativa para a refutação produzida. Assim, a visualização de (b) possui valor didático útil, através da comunicabilidade da estrutura da refutação em (a). 
Figura 1. Script para produção de refutação no ProofWeb

\begin{tabular}{|c|c|}
\hline 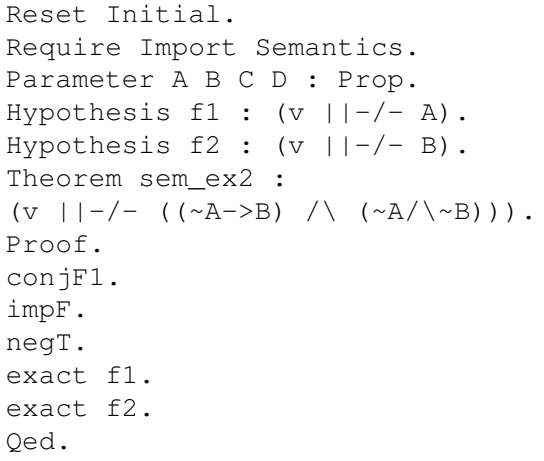 & 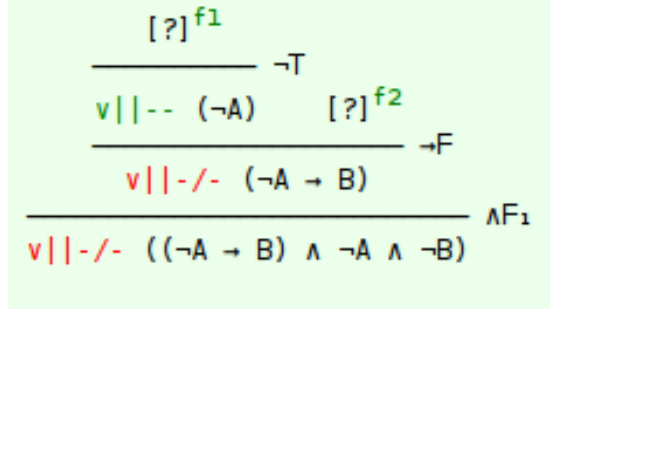 \\
\hline a) Script com sequência de táticas & b) Árvore resultante construída no P roofWeb \\
\hline
\end{tabular}

\section{O tutorial interativo TryLogic para Demonstrar ou Refutar}

O TRYLOGIC é um tutorial interativo desenvolvido a partir do TryOcam ${ }^{3}$ e do ProofWeb. O objetivo principal do TRYLOGIC é diminuir a sobrecarga cognitiva através de um tutorial passo-a-passo de forma sistemática, apresentado diferentes tópicos da lógica relacionados ao processo de DxR conjecturas lógicas. A ideia básica do projeto é fornecer um tutorial interativo para ensinar o aluno a solucionar problemas lógicos.

Figura 2. Lições do TRYLoGIC integradas ao Moodle

\section{Lógica Aplicada à Computação 2013.1}

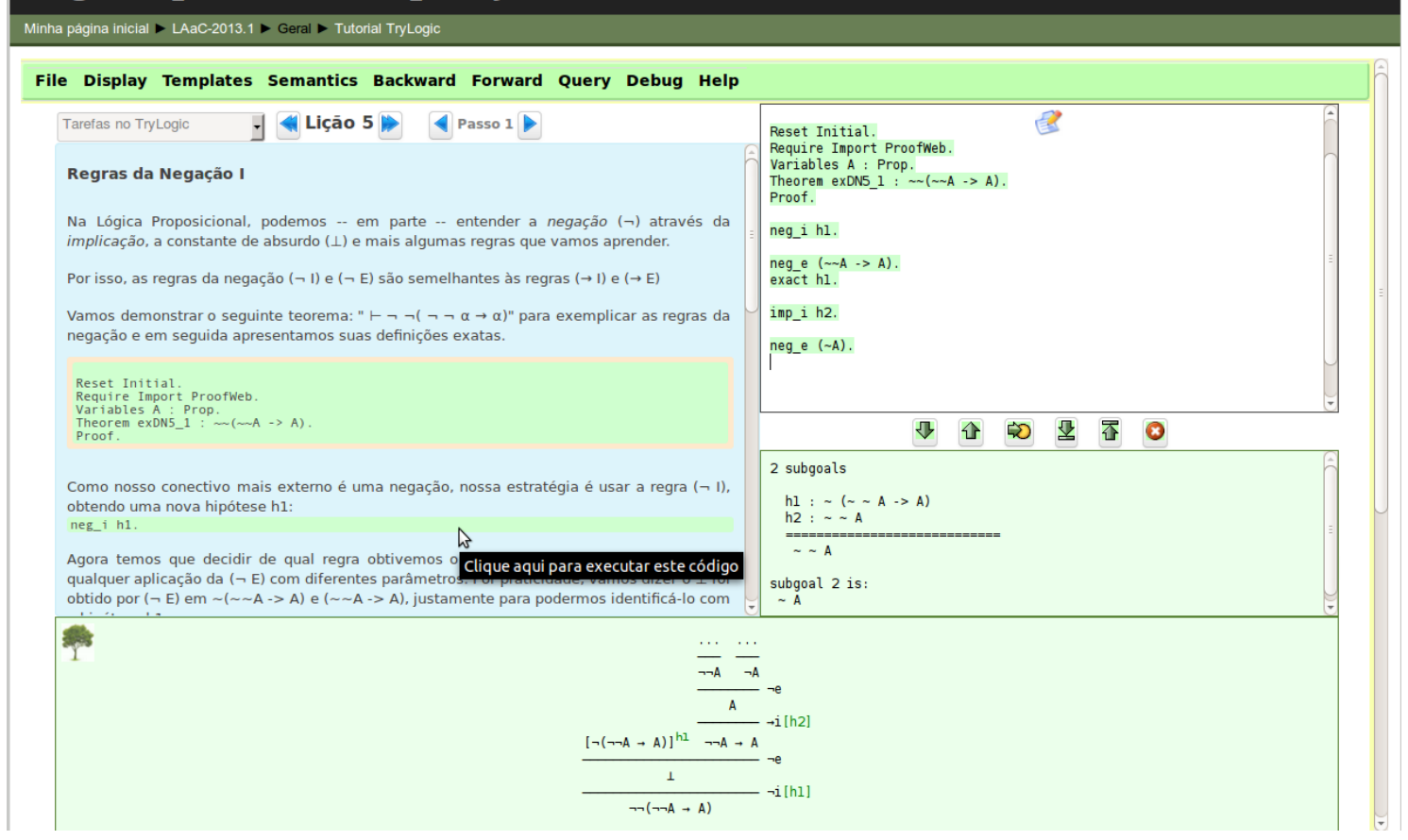

O Gerador de Conjecturas em Lógica Proposicional foi desenvolvido através da

\footnotetext{
${ }^{3}$ Disponível para acesso através do endereço http: //try . ocamlpro.com/
} 
colaboração de alunos de graduação bolsistas de iniciação científica ${ }^{4}$. A implementação foi realizada a partir de um gerador de fórmulas em comunicação com o SAT-solver $\mathrm{Limb} \circ \mathrm{l} \mathrm{e}^{5}$ para avaliar as fórmulas proposicionais. O Gerador de Conjecturas realiza a criação de conjecturas, no formato de exercício individualizado para o Coq, diretamente na área dos alunos no ProofWeb. Os alunos recebem cada tarefa ou conjectura em um template para demonstrar $(\Gamma \vdash \alpha)$ teoria $N_{p}$ e outro para refutar $(\Gamma \not \models \alpha)$ na teoria $\operatorname{Sem}_{p}$.

Com o objetivo de centralizar o uso das nossas ferramentas, nós investigamos um conjunto de alterativas de especificação da comunicação entre ferramentas de ensino, e de acordo com [Alario-Hoyos and Wilson 2010] e [Queirós and Leal 2012], uma das alternativas mais representativas para infraestruturas de integração entre plataformas de ensino é a IMS Learning Tools Interoperability ${ }^{6}$.

No nosso caso, a ferramenta provedora é o TRYLOGIC e a ferramenta consumidora pode ser qualquer plataforma de aprendizagem como o ATutor, Sakai e Moodle. O nosso objetivo através desta integração é fornecer uma infraestrutura genérica para a comunicação centralizada das nossas ferramentas (Gerador de Conjecturas, ProofWeb e TRYLOGIC) com qualquer uma plataforma de aprendizagem de nossa escolha que contenha uma implementação do IMS Basic LTI. A integração permite que um aluno cadastrado em sua plataforma, com nome de ferramenta consumidora, possua uma conta criada numa ferramenta provedora. Com o IMS LTI, o acesso ao TRYLOGIC é realizado pelos alunos e tutores no Moodle, pois as contas são criadas automaticamente no ProofWeb e no Moodle a partir do primeiro acesso dos usuários. O resultado da integração do TRYLOGIC é representado pelo diagrama de implantação na Figura 3.

Figura 3. Visão de implantação do TRYLoGIC com o Moodle (IMS LTI)

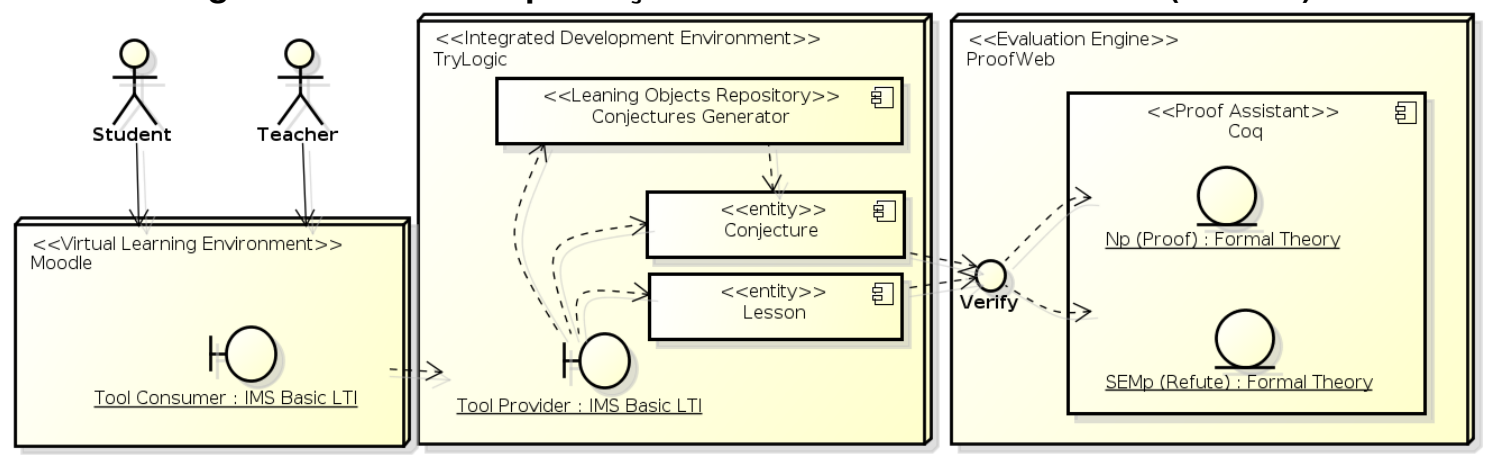

Dessa forma, qualquer colaborador que deseje utilizar o TRYLOGIC em qualquer plataforma como o Moodle poderá adicioná-la como ferramenta externa usando a nossa Launch URL, uma Consumer Key e uma Shared Secret cadastradas. Assim não é necessário instalar o TRYLOGIC, nem são necessárias permissões especiais do servidor.

Para realizar experimentos com a infraestrutura desenvolvida, nos semestres de 2012.2 e 2013.1 da disciplina de Lógica Aplicada à Computação de UFRN, nós utilizamos o TRYLOGIC para tarefas de demonstração e refutação. A tarefa de Demonstrar ou

\footnotetext{
${ }^{4}$ O Gerador de Conjecturas foi contribuição de alunos de iniciação científica Hudson Geovane e Elias Amaral, com o código disponível em http://lolita.dimap.ufrn.br/logicamente-ge/.

${ }^{5}$ Disponível em http: / / fmv. jku.at/limboole/.

${ }^{6}$ O IMS LTI foi desenvolvida em 2006 pela IMS Global Learning Consortium, e está disponível em http: / / www.imsglobal.org/lti/index.html.
} 
Refutar (DxR) foi atribuída a alunos da graduação que cursaram a disciplina de Lógica Aplicada à Computação (LAaC). Nosso maior desafio foi ensinar a resolução de tarefas de demonstrar ou refutar exclusivamente através do TRYLOGIC. Nas aulas presenciais ensinamos apenas os fundamentos teóricos. As experiências de aplicação serviram para verificar que o TRYLOGIC possibilita o entendimento do procedimento dedutivo no assistente de demonstração Coq para alunos que tiveram um breve contato teórico com o conteúdo de Dedução Natural para Lógica Proposicional. Assim, com o TrYLogiC o processo de experimentação via tentativa e erro é ensinado em um ambiente controlado.

\section{Considerações finais e trabalhos futuros}

Neste trabalho foi apresentada uma infraestrutura de convergência de ferramentas de ensino de Lógica com objetivo de: organizar em passo-a-passo a exposição dos conteúdos de Dedução Natural e Semântica Proposicional de forma sequencial e interativa; fornecer ao aluno tarefas interativas de autoavaliação; e integrar o Gerador de Conjecturas e TRYLOGIC com o Moodle via IMS Basic LTI. As nossas contribuições sobre o ensino de Lógica fazem parte de uma iniciativa que precisa ser aprimorada, em particular:

- a implementação de métricas de dificuldade para derivações para o Gerador de Conjecturas com o objetivo de garantir a existência de uma métrica efetiva; e

- a geração de conjecturas para a Lógica de Primeira Ordem com o objetivo de produzir novas tarefas e novas lições de Lógica de Primeira Ordem.

\section{Referências}

Alario-Hoyos, C. and Wilson, S. (2010). Comparison of the main alternatives to the integration of external tools in different platforms. In Proceedings of the International Conference of Education, Research and Innovation, ICERI, pages 3466-3476.

Barland, I., Felleisen, M., Fisler, K., Vardi, M. Y., and Kolaitis, P. (2000). Integrating Logic into the Computer Science Curriculum. Innovation and Technology in Computer Science Education.

Hendriks, M., Kaliszyk, C., Raamsdonk, F. V., and Wiedijk, F. (2010). Teaching logic using a state-of-the-art proof-assistant. Acta Didactica Napocensia, 3(2):35-48. Disponível em: <http://dppd.ubbcluj.ro/adn/article_3_2_4.pdf>.

Queirós, R. and Leal, J. P. (2012). Orchestration of e-learning services for automatic evaluation of programming exercises. Journal of Universal Computer Science, 18(11):1454-1482.

Sweller, J., Ayres, P., and Kalyuga, S. (2011). Cognitive Load Theory. Explorations in the Learning Sciences, Instructional Systems and Performance Technologies. Springer.

Terrematte, P., Costa, F., and ao Marcos, J. (2011). Logicamente: A Virtual Learning Environment for logic based on Learning Objects. In Third International Conference Tools for Teaching Logic., volume 6680 of Lecture Notes in Artificial Intelligence, pages 223-230. Springer-Verlag, Berlin. 